Research Article

\title{
Computer Network Dynamic Balance Flow Distribution Based on Closed-Loop Particle Swarm Feedback Model
}

\author{
Wei Zhou \\ Information Engineering Department, Suzhou University, Suzhou 234000, China \\ Correspondence should be addressed to Wei Zhou; weizhou@ahszu.edu.cn
}

Received 24 November 2021; Accepted 14 December 2021; Published 28 December 2021

Academic Editor: Gengxin Sun

Copyright (c) 2021 Wei Zhou. This is an open access article distributed under the Creative Commons Attribution License, which permits unrestricted use, distribution, and reproduction in any medium, provided the original work is properly cited.

\begin{abstract}
Based on the closed-loop particle swarm feedback model, this paper proposes a graphical method to analyze the stability of the computer network dynamic balance system. First, based on the second-order time delay system model of congestion control, the stability of the system is described by characteristic pseudopolynomials. Secondly, based on the inverse line, the stability of the system is verified by graphical analysis methods, and the PID controller parameter range that guarantees the stability of the system is obtained, and the relationship between the controller proportional gain boundary and the network characteristic parameters is analyzed. Then, based on the analysis of the basic particle swarm optimization algorithm, the particle swarm evolution formula is divided into two parts, its own factors and social factors, and the influence of each part on the evolution speed and position of the particle swarm is analyzed, and an improved particle swarm is proposed. Finally, according to the above analysis, we find the corresponding equation from the appropriate solution in turn, thereby designing a class of particle swarm optimization algorithm with fewer intermediate variables. In view of the system involved in the classical PID control parameter tuning method, the improved particle swarm algorithm is applied to the parameter tuning and optimization of the PID controller. During the experiment, the improved PSO-PID controller optimization algorithm was used in the random early detection algorithm of active queue management, the process of the improved algorithm was researched and designed, and the relevant performance of the improved algorithm was verified through simulation experiments.
\end{abstract}

\section{Introduction}

At present, Internet users are gradually increasing and network business traffic is becoming more and more complex. The continuous growth of network demand poses a very serious challenge to the load capacity of the network, so the network congestion problem has become increasingly severe [1]. Network congestion control is of great significance to the stability and fairness of network systems, and it is also a very important part of communication network research. At the same time, it is also an interdisciplinary research topic involving network communications, computer science, and automation control. Active queue management, as an effective supplement to the congestion control mechanism, is currently a hotspot in network congestion control research. Congestion control is a typical feedback control. Therefore, with the rise of intelligent algorithms in control theory, research on network congestion based on intelligent control theory has attracted widespread attention [2-6].

As the scale of the communication network becomes larger and larger, the number of network nodes is increasing, and the network business traffic is becoming more and more complex. The increasing demand poses a severe challenge to the load capacity of the network, and the problem of network congestion is becoming more and more serious [7-9]. Congestion control is of great significance for ensuring the robustness of network systems and maintaining high service quality. It is an extremely active and important part of current communication network research and is an interdisciplinary research topic involving communication networks, computer science, and automatic control. In a closed-loop control system, the sensor is an indispensable link for information acquisition. In the control system, once the sensor's 
performance deteriorates due to aging or other reasons, it will have a serious impact on the monitoring and control systems of the industrial control system and cause immeasurable losses. The traditional intelligent control theory is applied to the active queue management algorithm, and there are problems of obvious queue length fluctuations and large packet loss rate. In view of the above problems, this article focuses on the research and discussion of the application of particle swarm optimization algorithm in the active queue management algorithm [10-13].

This paper studies and analyzes the random early detection algorithm in the algorithm. Aiming at the problem of the parameters of greater sensitivity and lack of stability, an improved RED algorithm is analyzed and designed based on it and verified by simulation experiments. Combining the advantages of integral separation PID and incomplete differential PID, the working principle of the improved PID controller is analyzed, and the algorithm implementation steps and parameter tuning methods are explained. The first is a particle swarm optimization algorithm based on chaotic search to optimize system parameters. This method is based on the ergodicity of system nodes to improve the efficiency of the algorithm. The second is the particle swarm optimization and Broyden-Fletcher-Goldfarb-Shanno (BFGS) hybrid optimization algorithm, which combines the global search of the particle swarm optimization algorithm and the fast convergence characteristics of the BFGS method based on gradient optimization to improve the algorithm convergence speed and reduce the adverse effect on the system performance due to the integral saturation effect and the differential amplification effect on the noise in the traditional PID control.

\section{Related Work}

Most optimization problems arise from practice. Only when a certain type of problem accumulates to a certain extent and attracts the attention and interest of practical workers and theoretical researchers, will the research on these problems be promoted. Network congestion will lead to the probability of packet loss. The end-to-end network delay will increase, which will also lead to a decline in link utilization, a sharp drop in throughput, and even paralysis of the entire network system. In recent years, more and more scholars have begun to pay attention to and start to study the problem of network congestion, hoping to find ways and means to effectively solve such problems [14-17].

Babaeinesami [18] believes that the congestion control system is actually a time lag system, especially for the Internet and Wide Area Network (WAN). The active queue management algorithm applicable to (LAN) or Metropolitan Area Network (MAN) may not be able to work effectively in a wide area network environment with a large time delay. Kamil [19] believes that this kind of time delay cannot be ignored, and we can consider constructing a more effective congestion control algorithm with the help of time delay system control theory. Amin [20], on the basis of model fitting, applied the principle of internal model compensation, designed a robust delay compensation active queue management algorithm, used Smith predictor to compensate the delay, designed the controller according to the Dahlin algorithm, and proposed a prediction PI-controlled active queue management algorithm. Based on Lyapunov functional analysis of multivariable time delay systems, Guo [21] gave the robust stabilization conditions for the system to be exponentially stable with decay degree and proposed a robust P-control active queue management algorithm. Yousif [22] proposed a large time delay network congestion control algorithm based on an improved network model, taking into account the high-frequency component $\triangle(s)$ in the system model, analyzing the relationship between the characteristic roots of the closed-loop system and the characteristic polynomial coefficients, and designing PID controller parameters based on $D$ stability domain. The researchers applied numerical optimization methods to obtain the controller parameters for the constraint conditions of variable time delays, so that the characteristic roots of the closed-loop system fell within the $D$ stable region.

Researchers pointed out that inappropriate parameter configuration makes the system output easy to deviate from the control target, which will cause severe oscillations in the time delay network environment, thereby reducing the utilization of the bottleneck link and increasing the end-toend delay. Then use the usual local optimization method to get the precise position of the global extreme point. This is the most common way to transform the global optimization problem into a local optimization problem, and it is also an indirect method to solve such problems [23]. With the emergence of more and more complex optimization problems in industrial production and the gradual development of theoretical research, people have found that many optimization problems have properties that are different from local optimization problems. There are local extreme points, or the feasible region is neither a convex set nor a convex function. In some problems, the objective function cannot be expressed analytically. The function value can be obtained only through some procedures and steps. These problems are difficult to transform into local by the above methods. The researchers designed an immune PI controller in NCS. A PI controller based on immune algorithm is designed to reduce the impact of time delay on NCS performance, and Smith predictor is used to compensate for random time delay to further reduce its impact on system performance. This method makes the PID controller have the ability to predict the network delay, and the PID parameters are adjusted online according to the predicted output error at the future time, which greatly improves the control performance of the system and has strong engineering significance [24-27].

\section{Computer Network Dynamic Management}

3.1. Mathematical Analysis of Closed-Loop Particle Swarm. The principle of closed-loop particle swarm congestion algorithm is to design algorithms with superior performance to avoid network congestion. If the characteristics of the data flow can be determined and the network performance parameters are also available, an open-loop congestion control 
algorithm can be used. If the characteristics of the data flow cannot be determined, or the system cannot provide the reserved resources, a closed-loop congestion control algorithm can be used.

$$
\sum P(A, B)-\sum P(A \mid B) \sum P(B)=0
$$

In practical applications, due to the heterogeneity, complexity, and unpredictability of the Internet, closed-loop congestion control is mainly used. The closed-loop congestion control algorithm can dynamically adjust the parameters to adapt to the complex and changing network environment, but the performance of the algorithm will also be affected to a certain extent due to the increase of the feedback delay.

$$
\int P(A \mid B) \mathrm{d} A \mathrm{~d} B-P(B \mid A)^{*} \iint P(A) P(B) \mathrm{d} A \mathrm{~d} B=0 .
$$

According to the type of congestion control, the congestion control algorithm is divided into two categories: window congestion control and rate congestion control. The window congestion control algorithm controls the data flow in the network by adjusting the size of the TCP window. The rate congestion control algorithm is to obtain the relationship between throughput and network-related performance parameters through mathematical analysis and modeling of the TCP window control mechanism.

$$
\begin{aligned}
& \forall i, \\
& j=C(t, k), \\
& A(i) \cap A(j)-A(i, j)=\varnothing .
\end{aligned}
$$

The first part is the previous speed of the particles, which makes the particles have a tendency to expand in the search space, so that the algorithm has the ability to search globally; the second part is the "cognition" part, which represents the process of the particles absorbing their own experience and knowledge. The third part is the "social" part, which represents the process by which particles learn the experience of other particles and shows the sharing of information and social collaboration between particles.

3.2. Computer Network Control. When the network load is low, the throughput will increase linearly with the increase of the load, and the response time (RT) at this stage is in a slowly increasing stage; when the network load passes the knee point,

$$
\begin{aligned}
& \forall i, \\
& j=C(0,0), \\
& A \cup A(i) A(j)=0 .
\end{aligned}
$$

The throughput will increase slowly, and the response time will increase at a faster rate; when the network load crosses the cliff point, the throughput will begin to decrease sharply, and the response time will increase accordingly.
The main factors that cause network congestion include low processor capabilities of intermediate devices and fewer network bandwidth resources. These factors will directly lead to reduced network performance, sudden increase in packet loss probability, increased network delay, and reduced throughput, etc. The database uses programming to realize the connection with the management system of Figure 1 and the import and export of data, query, and other functions.

Using the average time delay trend theory to build NCS into a TS fuzzy model to approximate the system, this model has never obtained an acceptable solution when dealing with problems, because there is no connection between individuals, and a group of $m$ is equivalent to $m$ particles running alone.

The general strategy for constructing a global optimization method is usually based on the specific characteristics of the problem, so that the test point sequence obtained by the algorithm is as close as possible to the global minimum position of the function to be optimized. Without too much prior information about the function to be tested, the function to be optimized can only be uniformly covered by the detection point column, that is, the global strategy, as the calculation proceeds.

$$
R(i, j, t)=\sum A \cdot \mathrm{Gau}(s s) * \sum\left(A \cdot \mathrm{Gau}(s s) \cdot T_{h}\right)^{2} .
$$

Constraints are certain restrictions on independent variables when seeking the extreme value of the objective function. For example, in integer programming applications, all variables are required to be integers. In some resource planning problems, all variables required as resource quantities are all positive numbers. In addition, in some problems, the independent variables must also satisfy the basic equations and performance equations of the physical system.

3.3. Feedback Model Flow Calculation. At present, in the feedback model network transmission, compared with the circuit switching technology, the packet switching technology does improve the channel utilization. However, when a lot of data share a link, the message error phenomenon may occur, which will make the terminal more complex. The Internet mainly uses the TCP/IP communication protocol, which is a best effort service model, while the telecommunication network in Figure 2 uses an "acceptance control" network service mechanism.

Record the best result among the 100 experimental results, namely, the minimum fitness value and the average minimum fitness value of the 100 experimental results, as well as the standard deviation of the global minimum fitness change in the 100 experimental results. The minimum fitness value and the average minimum fitness value are used to measure the effect of the algorithm, and the standard deviation is used to measure the stability of the algorithm. Finally, draw the curve of the 


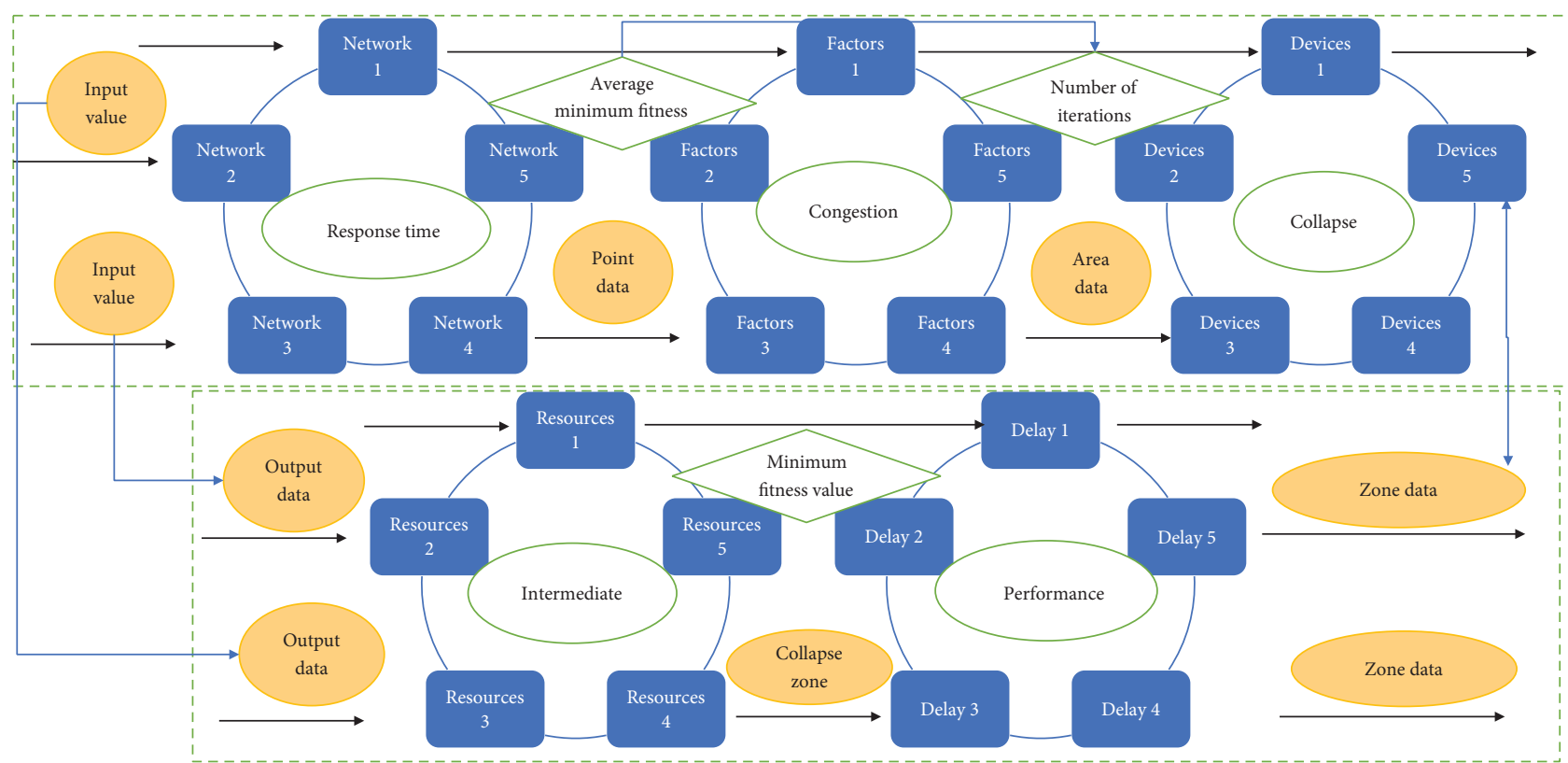

FIGURE 1: Computer network control topology.

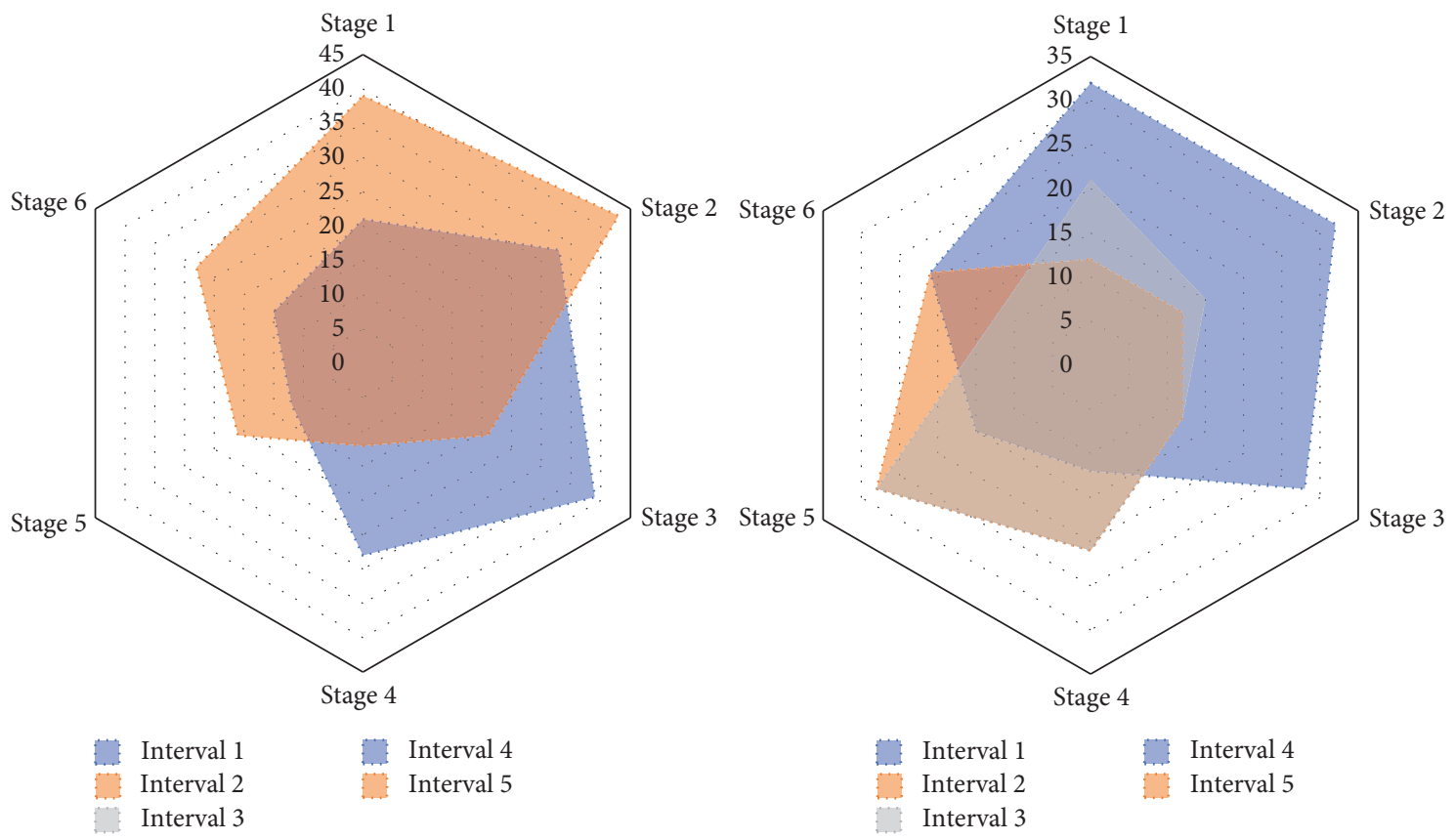

Figure 2: Feedback model traffic radar distribution. 
average minimum fitness value of each step in $100 \mathrm{ex}$ periments with the number of iterations.

$\sqrt{\left(A \cdot \operatorname{Gau}(s s) \cdot T_{w}\right)^{2}+\left(B \cdot \mathrm{Gau}(s s) \cdot T_{g}\right)^{2}}=\sqrt{(A+B) *(A-B)}$.

Once network resources are found to be lacking, if the mechanism of limiting the number of users is used to ensure that the network service quality of old users can no longer meet the current needs, the only way is to reduce the quality of service (QoS) of all users to meet the needs of users, but this will lead to network resource retransmission lost packets being more occupied by users, while the network will reduce the quality of service and discard packets, resulting in network congestion in a series of vicious cycles.

$$
\text { if } a, b, s s \in R(c, t) \text {, }
$$

$\sum \operatorname{Gau}(a, b ; s s)=\exp \left(-\frac{\left(a^{2}+b^{2}\right)}{2 s s^{2}}\right)+\exp \left(-\frac{\left(a^{2}-b^{2}\right)}{2 s s^{2}}\right)$

PID parameter tuning methods can generally be divided into two categories. The first type is based on the frequency domain index method, to meet the minimum gain margin and phase margin as the goal, which can obtain better robustness but may not obtain ideal time-domain characteristics. The other is a method based on time-domain indicators, which takes a certain performance indicator or the minimum weighted sum.

This type of method has better output response indicators, but it cannot guarantee better robustness. The relationship between the input and the output of the neuron model that adopts the probabilistic transfer function is uncertain, and a random function is needed to describe the probability that the output state is 1 or 0 .

\section{Construction of a Computer Network Dynamic Balance Flow Distribution Model}

4.1. Closed-Loop Particle Swarm Detection. If the performance of the closed-loop particle swarm processor is low or its performance is reduced due to other factors, its ability to process the buffer queue in the router will be reduced, which will cause data packets to wait for a long time, and the data sender will perform operations such as timeout and retransmission.

Further deepen network congestion. In practical applications, the quality and cost of routers will inevitably limit network resources, but the link capacity of network intermediate devices in Table 1, the performance of the processor, and the size of the cache all have growth limitations. Therefore, when the resources provided by the network cannot improve its service quality, how to effectively control the network load can also solve the network congestion.

But the prerequisite is that this parallel operation must be truly implemented on a computer or other dedicated
TABle 1: Network load node distribution.

\begin{tabular}{rccc}
\hline \multicolumn{2}{c}{ Features service } & Network load & $\begin{array}{c}\text { Network } \\
\text { congestion }\end{array}$ \\
\hline & Processor 1 & 0.91 & 0.95 \\
& Processor 2 & 0.83 & 0.78 \\
Processor 3 & 0.88 & 0.93 \\
Network accuracy & Processor 4 & 0.95 & 0.93 \\
& Processor 5 & 0.72 & 0.81 \\
& Processor 6 & 0.67 & 0.56 \\
Processor 7 & 0.71 & 0.78 \\
\hline
\end{tabular}

arithmetic unit. In fact, purely using the program cannot achieve real parallelism. If real parallel operation is not achieved, there is no essential difference in the calculation time between $m$ particles and $m$ chaotic searches. Therefore, this is not used in the following experiments.

The calculation results obtained each time will not be exactly the same, so the results of multiple experiments are used to evaluate the effect of the algorithm.

$$
\left\langle\begin{array}{l|c}
\frac{R(c, t)}{2 \pi s s^{2}} & \frac{-B \cdot \mathrm{Gau}(s s) \cdot T_{w}}{\sum T_{w} * B} \\
\frac{A \cdot \mathrm{Gau}(s s) \cdot T_{w}}{\sum T_{w} * A} & \frac{-\left(\left(a^{2}+b^{2}\right) / 2 T^{2}\right)}{2 \pi s s^{2}}-\frac{\left(a^{2}-b^{2}\right)}{s^{2}}
\end{array}\right\rangle=\left\langle\begin{array}{c|c}
1 & 0 \\
0 & -1
\end{array}\right\rangle .
$$

It can be seen that the size of the window determines the upper limit of the data that can be sent, but the sender does not necessarily have to send the data of the entire window size. The size of the window specified by the TCP protocol can be changed as needed.

Similar to the sending end with a sending window, there is also a receiving window at the receiving end. The nature of the function to be optimized will gradually become clear. At this time, various criteria, such as the average value of the function and information entropy, can be used to adjust the search strategy of detection points.

$T_{h}(w, t, k)=\sqrt{\cos \left(2 \pi w t^{2}\right) e^{-\left(t^{2} / t t^{2}\right)}+\sin \left(2 \pi w t^{2}\right) e^{-\left(t^{2} / t t^{2}\right)}}$.

It makes the algorithm have a strong exploration ability in the initial iteration of the iteration and can continuously search for new areas, and then the development ability is gradually enhanced, so that the algorithm can search around the best possible solution.

4.2. Feedback Model Factor Iteration. Error integration criterion ISTE optimal setting method is for a specific controlled object. If the controlled object is known, then it only needs to consider using this ISTE error integration criterion as the optimization of the objective function implementation parameters. 


$$
T_{t}(w, t, k, t t)=\sqrt{-\sin \left(2 \pi w k^{2}\right) e^{-\left(t^{2} / t t^{2}\right)}-\cos \left(2 \pi w k^{2}\right) e^{-\left(t^{2} / t t^{2}\right)}} .
$$

The parameters obtained by this method depend on algorithm being continuously optimized by another algorithm along with the information fed back during the calculation process. Unlike other methods, the parameters obtained are static. It interactively accepts various commands input by users and outputs calculation results. It provides an open integrated environment where users can run a large number of commands provided by the system, including numerical calculations and graphics drawing.

Therefore, the algorithm parameters obtained by this method can usually make the algorithm obtain better performance. The parameters in Figure 3 have been obtained through theoretical research or empirical analysis; otherwise it is difficult to predict the effect of the entire algorithm.

TCP provides a unified data service interface for upper-layer applications. The data of the application process is delivered to TCP through this interface, and TCP buffers the data and, when appropriate, takes out the appropriate data length to form a data segment and send it out.

TCP does not know the format and meaning of the transmitted data, nor does it perform any processing on the data. The TCP protocol just treats the data to be transmitted by the application as an unstructured bit stream that can be divided into bytes, which is also the flexibility of TCP.

$$
\prod T_{i}(i, j) \times \prod T_{i}(i) \times T_{i}(j)-\prod A(i+1, j)-A(i-1, j)=0 .
$$

The inertia weight represents the proportion of the original speed in the next iteration. Generally, if $w$ is larger, the algorithm has stronger global search ability, and if $w$ is smaller, the algorithm has stronger local search ability. Therefore, the general setting $w$ is continuously reduced as the calculation progresses, so that the algorithm has a better global search ability in the early stage of operation and a better local search ability in the final stage.

4.3. Computer Network Queue Sorting. If multiple packets in the computer network queue enter the same router port at the same time, more packets will be queued in the router's buffer. When the queue length is greater than the buffer size of the router port, if there are still packets arriving, the router will directly discard the newly arrived packets.

If the buffer area of the device is large enough, more packets can be accommodated, but the data sender does not receive the confirmation command sent by the router, so the data sender will perform a timeout retransmission operation to increase the router's buffer burden; it will take up too much cache space at the same time, deepening the degree of network congestion.

$$
\frac{\partial T_{j}(i, j)}{\partial \prod T_{i}(i, j)}=\frac{\partial A(i, j+1)-\partial A(i, j-1)}{\prod T_{i}(i) * T_{i}(j)} .
$$

The TCP sender data packet size is 1000 bytes. It is configured as a UDP sender, with a link capacity of $3 \mathrm{Mbps}$ and a transmission delay of $30 \mathrm{~ms}$, which generates simulated actual interference traffic, with a link capacity of $30 \mathrm{Mbps}$ and a transmission delay of $25 \mathrm{~ms}$. The capacity of other links is $30 \mathrm{Mbps}$, and the transmission delay is $30 \mathrm{~ms}$. The evaluation model based on the analytic hierarchy process can combine the relevant factors of the forecast.

When facing a specific application problem, we must first analyze the nature of the problem and then determine the network model according to the characteristics of the problem. Finally, through network simulation analysis, determine whether the network is suitable and whether it needs to be modified. The sampling frequency is $160 \mathrm{~Hz}$, and the buffer in the router is 1125 packets. The simulation in Table 2 lasted for $200 \mathrm{~s}$, and CBR interference began to occur at the UDP sender $100 \mathrm{~s}$ after the simulation started.

The communication network is always in dynamic changes during operation. The network characteristic parameters such as the number of TCP connections reflecting the network load, the link capacity reflecting the channel conditions, and the round-trip time reflecting the transmission delay and queuing delay are always dynamically changing.

That is, there is uncertainty in the network characteristic parameters. In the design of the classic active queue management algorithm, for simplicity, it is usually assumed that the network characteristic parameters are fixed. In the case of relatively small parameter perturbation, ignoring the parameter uncertainty will have a relatively small impact on system performance.

$$
\frac{\partial A(i, j+1)-\partial A(i, j-1)}{\partial A(i, j+1)+\partial A(i, j-1)}= \begin{cases}T_{i}(i, j=0), & \text { if }(i, j) \in R(i=0, j=1) \\ 0, & \text { if }(i=0, j=0)\end{cases}
$$

In this process, each step of the particle swarm optimization algorithm is nested with $\mathrm{m}$-step chaos optimization. Obviously, the speed of the algorithm has slowed down, but the advantage is that the convergence of the algorithm becomes independent of the parameters, and the user does not need to care about how the parameters should be 


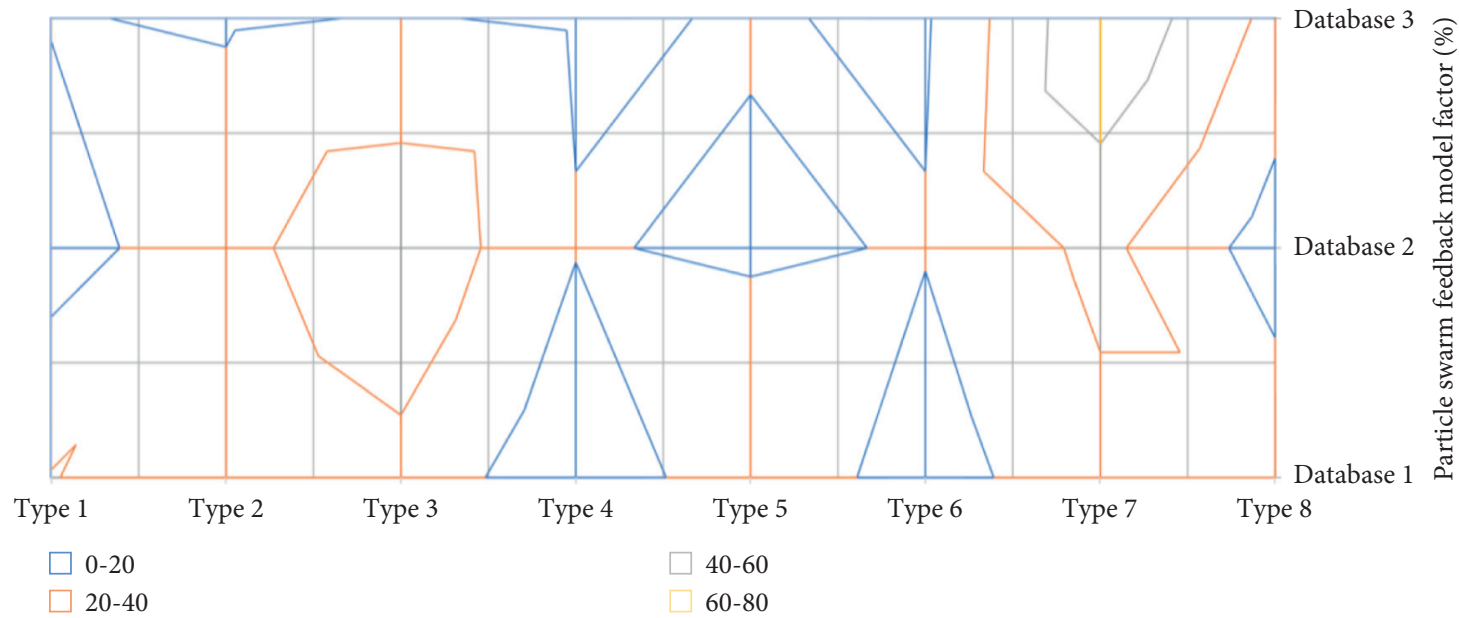

FIgURE 3: Factor distribution of particle swarm feedback model.

constructed and how to take their values. The entire parameter selection process becomes adaptive.

4.4. Performance Evaluation of Flow Distribution. The basic principle of traffic distribution performance is to monitor the average queue length in the router and use the average queue length as a signal of network congestion. When network congestion is detected, the corresponding link is selected to notify the data sender to appropriately reduce the sending rate. The best forecasting model is selected, and the forecast is made at the same time with the three indexes of forecast total, growth, and growth rate.

$\lim _{x \longrightarrow \infty}(T(i, j)+T(i-1, j-1))-\lim _{x \longrightarrow \infty}\left(\left(T_{i}(i, j)\right)^{2}+\left(T_{i}(i, j)\right)^{2}\right)^{(1 / 2)}=$.

The use of this software does not require a lot of computer knowledge, which is conducive to the use of professional designers. The design of the software is as humane as possible: the user enters data on the interface and sets the parameters, and due to the actual needs of production, there are a lot of input data, so the software allows calling the previous data files and proceeding on the basis of this data.

For the output signal of the control system, it is necessary to complete the function of controlling each device of the fully mechanized caving face, including the switch of each device and the shutdown processing when each device fails.

$$
\left\{\begin{array}{l}
w(i, j)=\sum_{d=1}^{n} i \times w_{d}(i, j), \\
w(i-1, j)=\sum_{d=1}^{n}(i-1) \times w_{d}(i-1, j-1), \\
w(i+1, j)=\sum_{d=1}^{n}(i+1) \times w_{d}(i+1, j+1) .
\end{array}\right.
$$

The process restriction relationship includes the relationship between physical balance and chemical balance, as well as the relationship between chemical reaction conversion rate and separation equipment efficiency. In addition, if a certain component is missing in a certain stream, the concentration of the component in the stream is equal to zero.

This is a restrictive relationship; the component concentration is proportional or the material flow is proportional; the flow divider divides the stream of Figure 4 into groups. If there are multiple streams of the same concentration, if one stream is divided into $S$ shares, and each stream has $\mathrm{C}$ components, the number of process restriction relations of the splitter is $S(C-1)$. Some process restriction relations are related to equipment performance, and such relations are also called equipment restriction relations.

When there are too many packets entering the same port of the router, the excess packets will be queued in the buffer area of the port for processing. If the packets accumulated in the buffer area exceed the capacity of the buffer area, the router will discard the later incoming packets. For incoming burst packets, it will cause batches of packets to be discarded.

When the buffer space is too large, the packet buffer delay will also be very large, causing the source to retransmit the packet due to timeout, and the router will still forward these timeout packets, thereby wasting network resources and increasing network congestion. After a few seconds of delay, the rear scraper must be turned off if the load is still overloaded. Otherwise, the system can be gradually restored to its original initial state.

\section{Application and Analysis of Computer Network Dynamic Balance Flow Distribution Model}

5.1. Network Dynamic Balance Data Processing. Research on network dynamic balance data shows that RED algorithm design parameters and network conditions make the performance of the algorithm very sensitive. Under certain network load conditions, many TCPs will still be 
TABLE 2: Description of computer network queue sorting.

\begin{tabular}{|c|c|c|c|c|c|}
\hline Network queue & Capacity 1 & Capacity 2 & Capacity 3 & Capacity 4 & Capacity 5 \\
\hline 1 & 99.75 & 30.41 & 57.67 & 60.55 & 43.70 \\
\hline 2 & 66.67 & 27.51 & 93.16 & 46.23 & 11.76 \\
\hline 3 & 56.92 & 23.10 & 6.19 & 52.64 & 32.07 \\
\hline 4 & 99.75 & 30.41 & 42.90 & 45.17 & 70.10 \\
\hline 5 & 28.67 & 18.31 & 83.38 & 43.57 & 16.53 \\
\hline
\end{tabular}

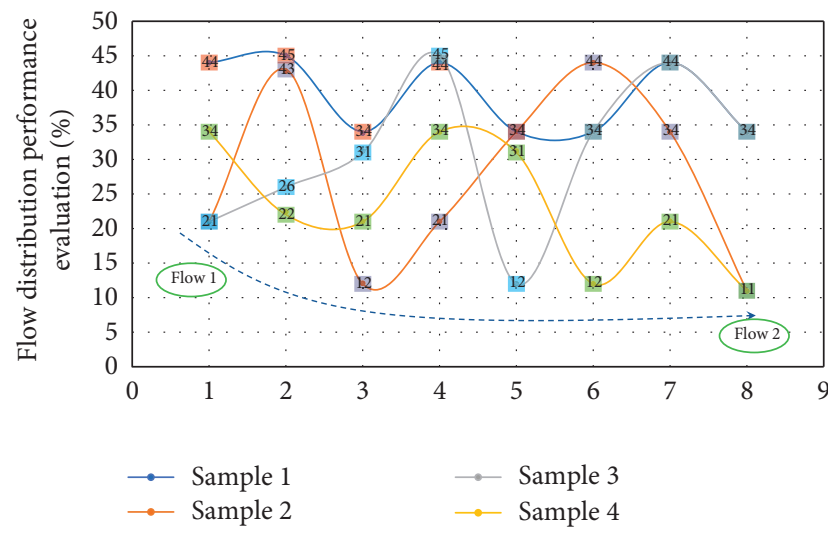

Figure 4: Flow distribution performance evaluation curve.

synchronized, resulting in reduced throughput, continuous queue oscillations, and delays.

Although the improved RED algorithm improves performance, it also increases the complexity of the algorithm. The RED algorithm does not consider that throughput and drop probability will be affected by the pair of packet sizes. Therefore, it will cause the RED algorithm to have a higher probability of packet loss for connections with a larger maximum transmission unit, resulting in fairness issues in network transmission.

$$
\begin{aligned}
& \exists X, \\
& X X \in\left(x-x^{2}=1\right), \\
& \operatorname{cov}(X)-E\left[X X^{T}-\left(E(X) E(X)^{T}\right)\right]=0 .
\end{aligned}
$$

This makes the forecast results more accurate and comprehensively reflect the changing laws of historical data. Let the control parameter $t$, which decreases its value with the algorithm progress, play the role of the annealing process temperature $T$; then for each value, the algorithm in Table 3 provides the expected output results for a given set of inputs. This set of known input and output data is called a training sample set.

If the set process conditions make the degree of freedom of the system zero, the only definite solution can be obtained mathematically. Whether this solution is meaningful in physics requires actual judgment. If the obtained solution is negative, it means that the preset variable value is unreasonable and needs to be reset.
The solution obtained must not only have physical meaning, but also be the best conditions for production. Therefore, it is necessary to set multiple sets of variable values and then compare the results to select the best set of variable values. This is an optimization problem of various design schemes, which is very important in design work.

5.2. Flow Distribution Model Simulation. Compared with the traditional drop tail algorithm, the random early detection algorithm of the traffic allocation model adds two mechanisms to queue management. First, the RED algorithm uses a probability determination mechanism instead of waiting for the queue to be full before discarding new arrivals. At the same time, the basis function neural network is used to fit the prediction results of these three indicators and related influencing factors.

Because some packets are discarded in advance, it can avoid the upcoming network congestion; second, the average queue length is used to calculate the probability of packet loss, instead of the instantaneous queue length, so it can absorb part of the short burst traffic as much as possible.

$$
\left\langle\begin{array}{l}
\lim _{x \rightarrow \infty} \operatorname{cov}(X)-\lim _{x \rightarrow \infty} \frac{n !}{r !}=0, \\
\lim _{x \rightarrow \infty} \operatorname{cov}(Y)-\lim _{x \rightarrow \infty} \frac{n !}{r !(n-r) !}=1 .
\end{array}\right.
$$

Then, draw a straight line and check the stable boundary of the proportional gain. According to the previous stability criterion, determine the stability boundary of the proportional gain. It can be seen that the stability boundary of the proportional gain in Figure 5 increases approximately in the same proportion as $\mathrm{N}$ increases. Similarly, $N=60$, go $=0.25 \mathrm{~s}$, C changes from 1500 to 5000 packets $/$, and the stability boundary is shown in the text.

If for each process, the given process conditions directly explain the numerical relationship between input materials and output materials, then the following block diagram model can be established to describe the material balance calculation; that is, the input materials of the $\mathrm{i}$-th process are $\mathrm{Xi}$ and $\mathrm{Yi}$. The output materials are $\mathrm{Xi}+1, \mathrm{Yi}+1$, and the relationship between the input and output materials is $t$, where $\mathrm{Xi}$ is the conversion factor of the material $\mathrm{Xi}$ in the $\mathrm{i}$-th process. 
TABLE 3: Optimization solution process of particle swarm combination.

\begin{tabular}{|c|c|c|}
\hline Steps number: & Algorithm solution process steps & For (int $i=0 ; i<\operatorname{sizeof}(\operatorname{srcnum}) / 4 ; i++$ ) \\
\hline 1 & The receiving end mechanism of the protocol & Int main () \\
\hline 2 & The returned load index code represents it & $\{$ Int $i, j$,low,high,mid,temp \\
\hline 3 & Copy from the header of the data & Int srcsum $[10]=\{10,91,8,71,61,5,41,3,21,1\}$ \\
\hline 4 & From packet to the ACK packet & for $(i=2 ; i<\operatorname{sizeof}(\operatorname{srcsum}) / 4 ; i++)$ \\
\hline 5 & The iMLCP sender is built for $T(i-1, j-1)$ & $\operatorname{srcsum}[\mathrm{i}]$ makes the degree of network congestion \\
\hline 6 & The same as the MLCP protocol & $\{$ temp $=\operatorname{srcsum}[\mathrm{i}]$ \\
\hline 7 & Similar to the MLCP protocol, it is $T_{i}(i, j)$ & low $=0 ;$ high $=\mathrm{i}-1 ;\}$ \\
\hline 8 & for $\left((x, y, z)^{2} \not \subset \varnothing\right)$ & while(low $\leq$ high) \\
\hline 9 & When packet loss occurs on the link & Public void bubbleSort(int arr[]) \\
\hline 10 & For item in queue $\lim _{x \rightarrow \infty}(n ! /(r !(n-r) !))$ & $\{$ mid $=($ low + high $) / 2 ;\}$ \\
\hline 11 & Its mechanism is the same as the TCP protocol & If $(\operatorname{srcsum}[\mathrm{mid}]>$ temp $)$ high $=$ mid -1 \\
\hline 12 & The 3 bits of the exponent are sorted & else $\operatorname{srcnum}[j+1]=$ temp \\
\hline 13 & The corresponding strategy on the TCP sender & low $=$ mid +1 \\
\hline 14 & The value of the minimum capacity field & For (int $i=0 ; i<\operatorname{sizeof}(\operatorname{srcnum}) / 4 ; i++$ ) \\
\hline 15 & It sends feedback based on the ACK packet & printf ("srcnum[\%d] = \%d",i,srcnum $[\mathrm{i}]$ ) \\
\hline
\end{tabular}

$$
\text { for }\left\{(x, y, z)^{2} \not \subset \varnothing\right\}, \int \operatorname{cov}(X) \operatorname{cov}(Y) \operatorname{cov}(Z) \mathrm{d} X \mathrm{~d} Y \mathrm{~d} Z=\oiiint r \sin \theta \cos \theta \mathrm{d} r \mathrm{~d} \theta
$$

From the physical meaning of process conditions, in actual production, it is inconvenient to obtain the numerical relationship between the input materials and output materials of each process. For example, for the separation process, in this process, the moisture content of the filter cake can be detected.

Therefore, for alumina Bayer process production, even if there is such a simple material balance calculation method in theory, using the numerical relationship between input materials and output materials in each process to calculate the material balance, it is impossible to obtain this in actual production.

5.3. Example Application and Analysis. The necessity criterion studies the range of controller parameters and analyzes the relationship between the proportional gain boundary and the network characteristic parameters. In the three-dimensional space, the parameter range of the PID controller that makes the TCP/AQM system stable is given. The comparison experiment and simulation experiment further verify the validity of the criterion.

$$
\left\|\begin{array}{cc}
\sqrt{b^{2}-4 a c} & \sin b \cos a \\
-\sin b \cos a & -\sqrt{b^{2}+4 a c}
\end{array}\right\|-\left\|\begin{array}{cc}
1 & -1 \\
-1 & 1
\end{array}\right\|=0 .
$$

The abnormality of the measurable variables of a certain subsystem is not only related to the measurable variables of the system, but also caused by the fault propagation of the measurable variables in the related subsystems.

$$
\lim _{x \rightarrow \infty} \frac{\partial w_{d}(i-1, j-1)}{\partial w_{d}(i, j-1)} \leq \frac{\sqrt{2} w_{d}(i, j)}{w(i, j)}, \quad \text { if }\{(i, j) \longrightarrow(1,-1)\} .
$$

The algorithm defines the weighted combination of the difference between the packet arrival rate and the link capacity and the difference between the instantaneous queue length and the target queue length as the link price. The learning system (neural network) can be based on the difference between the known output and the actual output (error signal).

The control goal of the REM algorithm is to match the packet arrival rate with the link capacity, so as to clear the buffer in the router, since the calculation of the packet arrival rate in Figure 6 has to adjust the system parameter values.

Analyzing the principle of the traditional PID controller, the integral link can eliminate the steady-state error, but when the system is greatly disturbed or the given value changes greatly, due to the large deviation, under the action of the integral link, there is integral saturation. The speed and accuracy of fitting are improved, and the problem of space load forecasting in mature large areas is better solved.

The output response produces larger overshoot and longer-term fluctuations. The differential link can improve the dynamic characteristics of the system, but the differential link has an amplifying effect on interference and noise, making the system particularly sensitive to interference.

$$
\sum P(S(i), S(j), \ldots, S(k) \mid T(t))=\frac{P(S(1), S(2), \ldots, S(k) \mid T(k))}{P\left(\sum S(i) \mid \sum T(t)\right)} \text {. }
$$

The Metropolis algorithm is executed at each value of $\mathrm{T}$. Therefore, the convergence speed is slow; especially when there are many variables, the objective function is more complicated, and the search space is relatively large, the time required to complete the calculation is simply unacceptable. If T0 is selected too large, the computer time will be too long. After that, the simplex method will extend the simplex in a certain direction to increase the step size. 


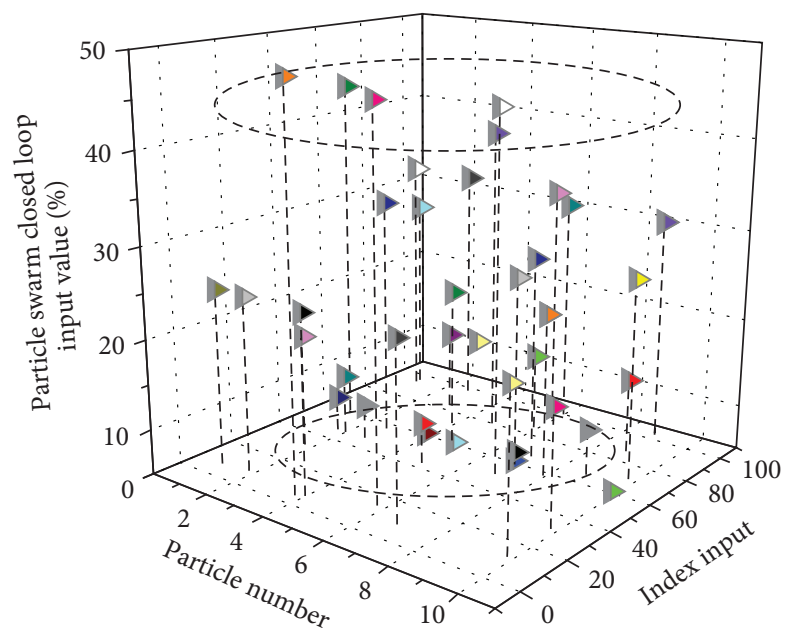

Figure 5: Particle swarm closed-loop input and output value distribution.

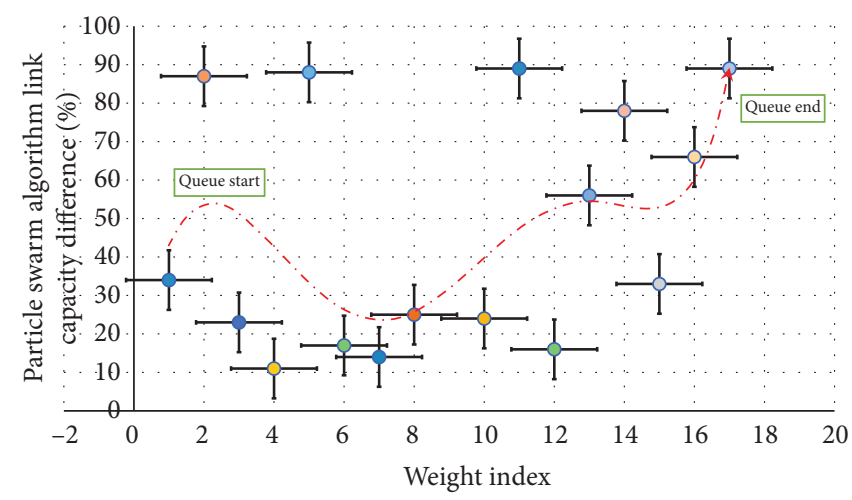

Figure 6: The distribution of the link capacity difference of the particle swarm algorithm.

$$
\left[\begin{array}{cc}
P(S(i)+S(k)) & P(S(i)) \\
P(S(k)) & P(S(i)-S(k))
\end{array}\right] \times\left[\begin{array}{cc}
P(S(i)) & -\sum T(t) \\
\sum T(t) & -P(S(k))
\end{array}\right]=\left[\begin{array}{cc}
S(i) & -\sin i \\
\cos i & T(i)
\end{array}\right] .
$$

When approaching the extreme point, the simplex will shrink laterally or from all directions and pull to the extreme point by itself. After the above steps, a new point is obtained. The new point and the original $\mathrm{N}$ vertices form a new simplex, and at least one of the vertices has a function value smaller than the original simplex, and the optimization is based on this. Repeated iterations can always shrink to the minimum value of the function.

\section{Conclusion}

Aiming at the time delay characteristics of the particle swarm congestion control model, this paper proposes an active queue management method based on robust control theory. First, for the discrete time delay model of the TCP/ AQM system, a bounded model of the TCP/AQM system feedback control is designed. Since the fully mechanized caving face system is very complex in function and structure, we adopt a multilevel and distributed system failure analysis method. Through the continuous feedback of the information about the function to be optimized during the calculation process, the local minimum is transformed into the global. At the same time, in order to improve the adaptability of the congestion control algorithm, an active queue management method based on intelligent control theory is proposed. The process characteristics of the Bayer process and main processes are analyzed, the characteristics and difficulties of the material balance calculation in actual production are analyzed, the principle of system modeling is proposed, and the mathematical model of the actual process of the Bayer process is established. This model is a mathematical abstraction of a nonlinear, multifeedback largescale complex production system, which contains all the information of the entire production process. All the above signals are used as the input of the fully mechanized caving control system to provide a basis for the control system to make a judgment. The experimental results show that, for the inaccuracy of the congestion control model, while 
considering the queue factor and load factor, the fuzzy control is used to design a fast-response active queue management algorithm, and the neural network control is used to design an adaptive active queue management algorithm. The congestion control model is time-varying, and particle swarm optimization technology can be used to optimize the design of an active queue management algorithm with self-tuning parameters.

\section{Data Availability}

The data used to support the findings of this study are available from the corresponding author upon request.

\section{Conflicts of Interest}

The authors declare that there are no conflicts of interest.

\section{Acknowledgments}

This work was supported by the Key Disciplines of Computer Science and Technology (2019xjzdxk1), Teaching Research Project (2018jyxm0960), and College of Modern Industry (szxy2021cyxy04).

\section{References}

[1] K. Deželak, P. Bracinik, K. Sredenšek, and S. Seme, "proportional-integral controllers performance of a grid-connected solar pv system with particle swarm optimization and ziegler-nichols tuning method," Energies, vol. 14, no. 9, p. 2516, 2021.

[2] K. Patne, N. Shukla, S. Kiridena, and M. K. Tiwari, "Solving closed-loop supply chain problems using game theoretic particle swarm optimisation," International Journal of Production Research, vol. 56, no. 17, pp. 5836-5853, 2018.

[3] H. Pan, W. Mi, X. Lei, and J. Deng, "A closed-loop brainmachine interface framework design for motor rehabilitation," Biomedical Signal Processing and Control, vol. 58, Article ID 101877, 2020.

[4] H. Lin, B. Zhao, D. Liu, and C. Alippi, "Data-based fault tolerant control for affine nonlinear systems through particle swarm optimized neural networks," IEEE/CAA Journal of Automatica Sinica, vol. 7, no. 4, pp. 954-964, 2020.

[5] M. Rahimi and H. Fazlollahtabar, "Optimization of a closed loop green supply chain using particle swarm and genetic algorithms," Jordan Journal of Mechanical \& Industrial Engineering, vol. 12, no. 2, 2018.

[6] J. F. Tuttle, R. Vesel, S. Alagarsamy, L. D. Blackburn, and K. Powell, "Sustainable NOx emission reduction at a coalfired power station through the use of online neural network modeling and particle swarm optimization," Control Engineering Practice, vol. 93, Article ID 104167, 2019.

[7] S. M. H. Mousakazemi, "Computational effort comparison of genetic algorithm and particle swarm optimization algorithms for the proportional-integral-derivative controller tuning of a pressurized water nuclear reactor," Annals of Nuclear Energy, vol. 136, Article ID 107019, 2020.

[8] M. I. Solihin, W. H. Lim, S. S. Tiang, and C. K. Ang, "Modified particle swarm optimization for robust anti-swing gantry crane controller tuning," in Lecture Notes in Electrical Engineering, vol. 666, pp. 1173-1192, Unmanned System Technology, 2021.
[9] D. S. Acharya, B. Sarkar, and D. Bharti, "A fractional order particle swarm optimization for tuning fractional order PID controller for magnetic levitation plant," Measurement, Instrumentation, Control and Automation, pp. 5-6, 2020.

[10] Z. Qi, Q. Shi, and H. Zhang, "Tuning of digital PID controllers using particle swarm optimization algorithm for a CAN-based DC motor subject to stochastic delays," IEEE Transactions on Industrial Electronics, vol. 67, no. 7, pp. 5637-5646, 2019.

[11] X. Xu, Z. Gong, W. Guo, Z. Wu, E. Herrera-Viedma, and F. J. Cabrerizo, "Optimization consensus modeling of a closed-loop carbon quota trading mechanism regarding revenue and fairness," Computers \& Industrial Engineering, vol. 161, Article ID 107611, 2021.

[12] J. Chen, M. N. Omidvar, M. Azad, and X. Yao, "Knowledgebased particle swarm optimization for PID controller tuning," in Proceedings of the 2017 IEEE Congress on Evolutionary Computation (CEC), pp. 1819-1826, Evolutionary Computation, Donostia, Spain, June 2017.

[13] J. Guo, H. Yu, and M. Gen, "Research on green closed-loop supply chain with the consideration of double subsidy in e-commerce environment," Computers \& Industrial Engineering, vol. 149, Article ID 106779, 2020.

[14] V. Hajipour, M. Tavana, D. Di Caprio, M. Akhgar, and Y. Jabbari, "An optimization model for traceable closed-loop supply chain networks," Applied Mathematical Modelling, vol. 71, pp. 673-699, 2019.

[15] T. J. Shima and H. A. Bashir, "Performance assessment of particle swarm optimisation and differential evolution algorithms on integral sliding mode control of cart-inverted pendulum," International Journal of Nonlinear Dynamics and Control, vol. 2, no. 1, pp. 16-18, 2021.

[16] M. Ryalat, H. Salim Damiri, and H. ElMoaqet, "Particle swarm optimization of a passivity-based controller for dynamic positioning of ships," Applied Sciences, vol. 10, no. 20, p. 7314, 2020.

[17] B. Sarkar, M. Tayyab, N. Kim, and M. S. Habib, "Optimal production delivery policies for supplier and manufacturer in a constrained closed-loop supply chain for returnable transport packaging through metaheuristic approach," Computers \& Industrial Engineering, vol. 135, pp. 987-1003, 2019.

[18] A. Babaeinesami, H. Tohidi, and S. M. Seyedaliakbar, "Designing a data-driven leagile sustainable closed-loop supply chain network," International Journal of Management Science and Engineering Management, vol. 16, no. 1, pp. 14-26, 2021.

[19] H. G. Kamil, O. T. Makki, and H. M. Umran, "Optimal tuning of a linear quadratic regulator for position control using particle swarm optimisation," IOP Conference Series: Materials Science and Engineering, vol. 671, no. 1, Article ID 012047, 2020.

[20] S. H. Amin, G. Zhang, and M. N. Eldali, "A review of closedloop supply chain models," Journal of Digital Information Management, vol. 2, no. 4, pp. 279-307, 2020.

[21] J. Guo, L. He, and M. Gen, "Optimal strategies for the closedloop supply chain with the consideration of supply disruption and subsidy policy," Computers \& Industrial Engineering, vol. 128, pp. 886-893, 2019.

[22] S. Y. Yousif and M. J. Mohamed, "Design of robust FOPIFOPD controller for maglev system using particle swarm optimization," Engineering and Technology Journal, vol. 39, no. 4A, pp. 663-667, 2021.

[23] S. Vadi, F. B. Gurbuz, S. Sagiroglu, and R. Bayindir, "Optimization of pi based buck-boost converter by particle swarm optimization algorithm," in Proceedings of the 2021 9th 
International Conference on Smart Grid (icSmartGrid), pp. 295-301, Setubal, Portugal, June 2021.

[24] A. T. Azar, F. E. Serrano, and S. Vaidyanathan, "Proportional integral loop shaping control design with particle swarm optimization tuning," Advances in System Dynamics and Control, pp. 24-57, 2018.

[25] S. Saraeian, B. Shirazi, and H. Motameni, "Adaptive control of criticality infrastructure in automatic closed-loop supply chain considering uncertainty," International Journal of Critical Infrastructure Protection, vol. 25, pp. 102-124, 2019.

[26] J. Ghahremani-Nahr, H. Nozari, and S. E. Najafi, "Design a green closed loop supply chain network by considering discount under uncertainty," Journal of Applied Research on Industrial Engineering, vol. 7, no. 3, pp. 238-266, 2020.

[27] T. A. Khan, K. Ullah, G. Hafeez et al., "Closed-loop elastic demand control under dynamic pricing program in smart microgrid using super twisting sliding mode controller," Sensors, vol. 20, no. 16, p. 4376, 2020. 